\section{Determinantes precoces en el desarrollo de injuria renal aguda durante la sepsis abdominal experimental}

\author{
TOMÁS REGUEIRA PHD ${ }^{1}$, MAX ANDRESEN MSC ${ }^{1}$, \\ MARCELO MERCADO ${ }^{1}$, FELIPE LILLO $^{2, a}$, DAGOBERTO SOTO PHD ${ }^{1, b}$
}

\section{Early determinants of acute kidney injury during experimental intra-abdominal sepsis}

Background: Sepsis-induced acute kidney injury (AKI) is an early and frequent organ dysfunction, associated with increased mortality. Aim: To evaluate the impact of macrohemodynamic and microcirculatory changes on renal function and histology during an experimental model of intra-abdominal sepsis. Material and Methods: In 18 anaesthetized pigs, catheters were installed to measure hemodynamic parameters in the carotid, right renal and pulmonary arteries. After baseline assessment and stabilization, animals were randomly divided to receive and intra-abdominal infusion of autologous feces or saline. Animals were observed for 18 hours thereafter. Results: In all septic animals, serum lactate levels increased, but only eight developed AKI (66\%). These animals had higher creatinine and interleukin-6 levels, lower inulin and paraaminohippurate clearance (decreased glomerular filtration and renal plasma flow), and a negative lactate uptake. Septic animals with AKI had lower values of mean end arterial pressure, renal blood flow and kidney perfusion pressure, with an associated increase in kidney oxygen extraction. No tubular necrosis was observed in kidney histology. Conclusions: The reduction in renal blood flow and renal perfusion pressure were the main mechanisms associated with AKI, but were not associated with necrosis. Probably other mechanisms, such as microcirculatory vasoconstriction and inflammation also contributes to AKI development.

(Rev Med Chile 2014; 142: 551-558)

Key words: Acute kidney injury; Renal circulation; Sepsis.
1Departamento Medicina Intensiva, Facultad de Medicina, Pontificia Universidad Católica de Chile, Santiago, Chile. ¿Unidad de Medicina y Patología Comparada, Escuela de Medicina Veterinaria, Facultad de Ecología y Recursos Naturales, Universidad Andrés Bello, Santiago, Chile. ${ }^{a}$ Médico Veterinario. bBioquímico.

Fuente de apoyo financiero: Fondecyt 11100247.

Recibido el 23 septiembre de 2013, aceptado el 7 de mayo de 2014.

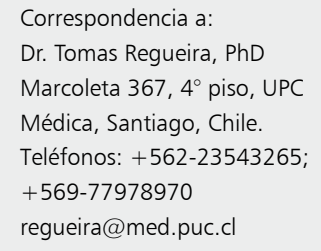

L a sepsis corresponde al conjunto de manifestaciones clínicas complejas secundarias a una excesiva y desregulada respuesta inmune del huésped a la infección ${ }^{1}$. El síndrome de disfunción multiorgánica (SDOM) es la principal causa de muerte en los pacientes sépticos ${ }^{2}$, siendo la injuria renal aguda (IRA) la disfunción orgánica más precoz y frecuente durante la sepsis, y que se asocia con una mayor mortalidad ${ }^{3}$.

Varios mecanismos han sido propuestos para explicar la génesis de la IRA en la sepsis, sin embargo, los procesos fisiopatológicos predominantes de la IRA durante la sepsis aún no son $\operatorname{claros}^{4-6}$. Clásicamente, la reducción en el flujo sanguíneo renal (FSR) se ha propuesto como el mecanismo más importante en la patogenia de la IRA ${ }^{5}$, sin embargo, la escasa presencia de necrosis reportada en tejido renal de pacientes que fallecen en sepsis con IRA hacen que la pérdida de flujo renal sea un proceso probablemente poco relevante $e^{7,8}$. Trabajos clínicos y experimentales recientes enfatizan la importancia de otros mecanismos en la génesis de la IRA en la sepsis, entre estos destaca la intensidad del cuadro inflamatorio y las alteraciones en la 
microcirculación más que los cambios globales en el FSR ${ }^{9-11}$.

El objetivo de este trabajo es evaluar el impacto de los cambios macro-hemodinámicos y del flujo sanguíneo y plasmático renal sobre la función e histología renal en un modelo experimental de sepsis intra-abdominal.

Nuestra hipótesis es que durante las primeras horas de la sepsis de origen abdominal, aquellos animales que desarrollan IRA tienen como mecanismo predominante alteraciones de la microcirculación renal por sobre cambios macro hemodinámicos significativos.

\section{Material y Métodos}

El presente estudio fue aprobado por el comité de ética y cuidado animal de la Facultad de Medicina de la Pontificia Universidad Católica de Chile.

\section{Preparación animal y configuración experimental}

Se utilizaron cerdos hembras con un peso promedio $30 \mathrm{~kg} \pm 3 \mathrm{~kg}$ que fueron mantenidos en ayuno, aunque con agua ad libitum desde la noche anterior al procedimiento experimental. Los animales fueron anestesiados con una dosis de pentobarbital, intubados y ventilados en modo control volumen con PEEP de $5 \mathrm{cmH}_{2} \mathrm{O}$ (Servo ventilator $900 \mathrm{C}$; Siemens-Elema ${ }^{\oplus}$, Solna, Suecia). $\mathrm{La} \mathrm{FiO}_{2}$ fue ajustada para mantener un nivel $\mathrm{paO}_{2}$ entre $100-150 \mathrm{mmHg}$. El volumen tidal fue de $10 \mathrm{ml} / \mathrm{kg}$ y ajustado para mantener un nivel de $\mathrm{paCO}_{2}$ entre 34-41 mmHg. Para la cirugía se utilizó anestesia con fentanilo $(30 \mu \mathrm{g} / \mathrm{kg} / \mathrm{h})$, midazolam $(1-2 \mathrm{mg} / \mathrm{kg} / \mathrm{h})$ y pancuronio $(1 \mathrm{mg} /$ $\mathrm{kg} / \mathrm{h}$ ). Durante la cirugía los animales recibieron solución salina $(0,9 \% \mathrm{NaCl} ; 10 \mathrm{ml} / \mathrm{kg} / \mathrm{h})$ para evitar hipovolemia. La temperatura corporal de los animales fue mantenida a $38 \pm 0,5^{\circ} \mathrm{C}$ con el uso de fluidos precalentados. Se instalaron catéteres para registro de presión y toma de muestras sanguíneas en las arterias carótida, renal derecha y pulmonar, mientras que catéteres venosos fueron instalados en las venasyugular y renal derecha. Se instaló flujómetro Doppler de ultrasonido (Transonic ${ }^{\varpi}$ System Inc., Ithaca, NY, USA) en la arteria renal derecha. Un catéter de drenaje urinario fue insertado en la vejiga. Finalmente, antes de cerrar la laparotomía, dos tubos de diámetro amplio
(Ch 32) fueron puestos con sus extremos en la cavidad peritoneal. Luego de la cirugía, la infusión de fentanilo se redujo a una dosis de mantención (sedación) de $5 \mu \mathrm{g} / \mathrm{kg} / \mathrm{h}$, sin alterar la infusión de midazolam ni de pancuronio. También se redujo la infusión salina a $5 \mathrm{ml} / \mathrm{kg} / \mathrm{h}$. Una vez comenzado el experimento, la manipulación fue evitada en orden a reducir la posibilidad del desplazamiento de los sensores instalados.

\section{Registro}

Se registraron los parámetros hemodinámicos sistémicos y regionales como frecuencia cardiaca, presiones arteriales de arteria carotidea y pulmonar, presión venosa central y renal derecha. Se registró también flujo de arteria renal derecha, gasto cardiaco (termodilución, promedio de 3 bolos a $4^{\circ} \mathrm{C} ; \mathrm{S} / 5$ Compact Critical Care Monitor; Datex-Ohmeda ${ }^{\circledR}$, Helsinki, Finlandia) y la presión de oclusión de la arteria pulmonar (PAOP). Las variables respiratorias $\left(\mathrm{FiO}_{2}\right.$ (fracción de oxígeno inspirado), presión meseta y compliance estática) fueron también registrados. Adicionalmente, la temperatura central fuemedida desde el sensor de temperatura ubicado en el catéter puesto en la arteria pulmonar $\left(\mathrm{CCO} / \mathrm{SvO}_{2}\right.$ catheter; Edwards Lifesciences, Munich, Alemania).

\section{Protocolo experimental}

Posterior a la cirugía, se permitió una hora de estabilización hemodinámica. Los animales fueron aleatoriamente dispuestos en dos grupos de experimentación de $18 \mathrm{~h}$. Un grupo control (C) (Animales operados, pero sin inducción de sepsis $)(n=6)$ y un grupo séptico $(n=12)$. Se indujo sepsis por vía intra-abdominal mediante la introducción de heces autólogas $(1 \mathrm{~g} / \mathrm{kg}$ de peso corporal) suspendidas en $200 \mathrm{ml}$ de solución salina calentada $\left(38^{\circ} \mathrm{C}\right)$, que fueron administradas a la cavidad abdominal mediante los tubos de diámetro amplio antes descritos. El grupo control recibió $200 \mathrm{ml}$ de solución salina tibia sin heces (placebo abdominal). Este modelo de sepsis severa porcina comprende resucitación por adición de fluidos en ausencia de vasopresores. Los objetivos hemodinámicos fueron mantener una presión arterial media (PAM) por sobre $60 \mathrm{mmHg}$ y una presión de capilar entre 8 y $10 \mathrm{mmHg}$. Para cumplir dichos objetivos, además de la infusión de solución salina programada $(5 \mathrm{ml} / \mathrm{kg} / \mathrm{h})$, bolos adicionales de 150 cc de solución salina fueron administrados 
según necesidad. Los animales también recibieron ceftriaxona (dosis única de $1 \mathrm{~g}$ ) y metronidazol (500 mg cada $8 \mathrm{~h}$ ) comenzando $2 \mathrm{~h}$ después de la inducción de la sepsis. Glucosa (50\%) fue administrada con el fin de mantener una glicemia entre 3,5-6 mM. Al final de los experimentos los animales fueron sacrificados con una sobredosis de potasio bajo anestesia profunda.

\section{Muestras sanguíneas}

Muestras de sangre para determinar contenido de hemoglobina, gases, lactato y creatinina, fueron colectadas al comienzo del procedimiento y luego de $18 \mathrm{~h}$ desde la arteria pulmonar y carótida, así como desde la vena renal y procesados de inmediato (i-STAT1 analyzer, Abbott, IL 60064, USA).

\section{Cálculos y definiciones}

Flujo sanguíneo renal indexado (FSRi): Flujo total renal derecho $(\mathrm{ml} / \mathrm{min}) /$ peso $(\mathrm{kg})$.

Flujo sanguíneo renal fraccional: Flujo total renal derecho $(\mathrm{ml} / \mathrm{min}) / \mathrm{GC}(\mathrm{ml} / \mathrm{min}) \times 100$.

Contenido arterial de oxígeno $\left(\mathrm{CaO}_{2}\right):(1,34 \mathrm{x}$ $\left.\mathrm{SaO}_{2} \mathrm{x}([\mathrm{Hb}] \times \mathrm{10})\right)+\left(0,031 \times \mathrm{PaO}_{2}\right)(\mathrm{ml} / \mathrm{l})$.

Transporte de oxígeno $\left(\mathrm{DO}_{2}\right)$ : Gasto cardiaco $(\mathrm{l} / \mathrm{min}) \times \mathrm{CaO}_{2}(\mathrm{ml} / \mathrm{l}) /$ peso $(\mathrm{kg})$.

$\mathrm{DO}_{2}$ renal: FSRi $\times \mathrm{CaO}_{2}$.

Consumo de oxígeno $\left(\mathrm{VO}_{2}\right)$ : Gasto cardiaco $(\mathrm{l} / \mathrm{min}) \times\left(\mathrm{CaO}_{2}-\mathrm{CvO}_{2}\right) /$ peso $(\mathrm{kg})$

$\mathrm{VO}_{2}$ renal: FSRi x $\left(\mathrm{CaO}_{2}\right.$ - Contenido venoso renal de $\mathrm{O}_{2}$ ).

Aclaramiento de lactato: (Lactato arterial Lactato vena renal) x FSRi.

Se definió que un animal estaba cursando IRA si sus niveles plasmáticos de lipocalina asociada a gelatinasa de neutrofilos (NGAL) al final del experimento eran iguales o superiores a $400 \mathrm{ng} /$ $\mathrm{ml}$, punto de corte validado en la literatura ${ }^{12-14}$ (Bio Porto Diagnosis, DK-2820, Dinamarca). No se utilizaron los niveles de creatinina ya que la elevación de esta puede ocurrir posterior a las $18 \mathrm{~h}$ (tiempo experimental en este diseño) ${ }^{15}$. Se midió también la presión de la vena renal derecha con el objeto de estimar la congestión y utilizada para calcular la resistencia vascular. Al final del experimento, se colecto muestra de sangre venosa para determinar el nivel de interleuquina-6 (IL-6) plasmática con el objeto de determinar la intensidad de la inflamación.

Durante las últimas tres horas del experimento se infundieron inulina (Inutest 25\%, Fresenius Kabi Austria GmbH, A-8055 Graz, Austria) (45 mg/kg bolo y $1.200 \mathrm{mg} / \mathrm{h}$ infusión) y paraaminohipurato (Merck \& Co, NJ 08889, USA) (18 mg/kg bolo y $720 \mathrm{mg} / \mathrm{h}$ infusión). Se calculó el aclaramiento de inulina y de $\mathrm{PAH}$, lo que permite estimar la tasa de filtración glomerular y el flujo plasmático renal (FPR), respectivamente. Se calculó la fracción de filtración a partir de estos parámetros. Finalmente, a partir del aclaramiento de PAH se calculo el FSR (aclaramiento de PAH indexado por peso/(1-hematocrito)) y se correlacionó con el FSR medido en la arteria renal derecha por doppler multiplicado por 2 (para aproximarse al flujo de ambos riñones).

Al final del experimento, el riñón izquierdo fue extraído para tinción con hematoxilina eosina y se realizó un análisis semicuantitativo del daño tisular renal en forma ciega por un nefro-patólogo experimentado.

\section{Estadística}

Una vez terminados los experimentos, se formaron 3 grupos para analizar, los animales control, los sépticos sin IRA y los sépticos con IRA. Para comparar los 3 grupos en el tiempo se utilizó un modelo lineal general de medidas repetidas, utilizando un factor intra sujetos (tiempo), un factor inter-sujetos (los 3 grupos antes mencionados) y una variable dependiente (la variable a analizar, e.g. lactato). Cuando los 3 grupos se compararon en un solo punto temporal, se utilizó ANOVA de un factor. Cuando se compararon 2 grupos (control vs sépticos) en un punto temporal, se utilizó prueba $\mathrm{T}$ para muestras independientes. Los datos son presentados como la media \pm desviación estándar; $\mathrm{p}<0,05$ fue considerado significativo. El software SPSS 16.0 (SPSS Inc. ${ }^{\circledR}$, Chicago, IL, USA) fue usado para los análisis estadísticos.

\section{Resultados}

\section{Función renal}

De los 12 animales sépticos, 8 desarrollaron IRA $(66 \%)$ de acuerdo a sus valores de NGAL. Ningún animal del grupo control desarrolló IRA (C: 40,2 $\pm 30 \mathrm{ng} / \mathrm{ml}$, sepsis sin IRA: $61,6 \pm 18 \mathrm{ng} /$ $\mathrm{ml}$, sepsis con IRA: $541 \pm 88 ; \mathrm{p}<0,001)$.

Los animales sépticos con IRA aumentaron significativamente sus niveles de creatinina respecto 
de los animales control y de los sépticos sin IRA $(\mathrm{p}=0,008)$ (Tabla 1). Del mismo modo, el aclaramiento de inulina durante las tres últimas horas del experimento fue significativamente menor en los animales sépticos con IRA (C: $85,9 \pm 10 \mathrm{ml} /$ min, sepsis sin IRA: $76,6 \pm 13 \mathrm{ml} / \mathrm{min}$, sepsis con IRA: $39,9 \pm 21 \mathrm{ml} / \mathrm{min} ; \mathrm{p}=0,01)$. Por otro lado, los riñones de los animales sépticos con IRA, a diferencia de los animales control y sépticos sin IRA, al final del experimento tuvieron una captación de lactato negativa, es decir, se volvieron productores de lactato (Tabla 1).

\section{Hemodinamia sistémica y regional}

Los animales sépticos recibieron más volumen que los animales control (C: 7,7 $\pm 0,9$ vs sépticos:
$11,4 \pm 1,2 \mathrm{ml} / \mathrm{kg} / \mathrm{h} ; \mathrm{p}=0,02)$, pero las presiones capilares pulmonares fueron similares al final del experimento $(\mathrm{p}=0,3)$ (Tabla 2). La PAM se mantuvo estable y sobre $60 \mathrm{mmHg}$ en todos los animales, pero fue menor en los animales sépticos con IRA al final del experimento $(\mathrm{p}=0,028)(\mathrm{Ta}-$ bla 2). Los niveles de lactato arterial aumentaron en ambos grupos sépticos, pero de mayor manera en los animales sépticos con IRA ( $\mathrm{p}=0,043)(\mathrm{Ta}-$ bla 2). Tanto el gasto cardiaco $(\mathrm{p}=0,3)$, como el transporte de oxígeno $(\mathrm{p}=0,5)$ y el consumo de oxígeno sistémicos $(\mathrm{p}=0,2)$ se mantuvieron estables y sin diferencias entre los grupos (Tabla 2).

Los valores finales de FSR indexado $(\mathrm{p}=0,048)$ $y$ fraccional $(p=0,04)$ fueron menores en el grupo séptico con IRA que en controles y que en animales

Tabla 1. Parámetros de función y hemodinamia renal

\begin{tabular}{|c|c|c|c|c|c|}
\hline Parámetro & Grupo & $\mathbf{n}$ & Basal & $18 \mathrm{~h}$ & Valor-p \\
\hline Creatinina $(\mathrm{mg} / \mathrm{dl})$ & $\begin{array}{l}\text { Control } \\
\text { Sepsis s/IRA } \\
\text { Sepsis c/IRA }\end{array}$ & $\begin{array}{l}6 \\
4 \\
8\end{array}$ & $\begin{array}{l}1,0 \pm 0,44 \\
0,9 \pm 0,20 \\
1,1 \pm 0,30\end{array}$ & $\begin{array}{l}1,2 \pm 0,40 \\
1,3 \pm 0,17 \\
1,9 \pm 0,34\end{array}$ & 0,008 \\
\hline Captación renal de lactato (umol/min/kg) & $\begin{array}{l}\text { Control } \\
\text { Sepsis s/IRA } \\
\text { Sepsis c/IRA }\end{array}$ & $\begin{array}{l}6 \\
4 \\
8\end{array}$ & $\begin{array}{l}0,94 \pm 0,6 \\
0,54 \pm 0,3 \\
0,64 \pm 0,8\end{array}$ & $\begin{array}{c}0,43 \pm 0,2 \\
0,9 \pm 0,5 \\
-0,57 \pm 0,4^{*}\end{array}$ & 0,16 \\
\hline Flujo sanguíneo renal indexado (ml/min/kg) & $\begin{array}{l}\text { Control } \\
\text { Sepsis s/IRA } \\
\text { Sepsis c/IRA }\end{array}$ & $\begin{array}{l}6 \\
4 \\
8\end{array}$ & $\begin{array}{l}4,8 \pm 0,9 \\
5,2 \pm 0,7 \\
4,1 \pm 1,2\end{array}$ & $\begin{array}{c}5,2 \pm 2,3 \\
5,2 \pm 1,3 \\
2,8 \pm 1,8^{*}\end{array}$ & 0,2 \\
\hline Flujo sanguíneo renal fraccional (\%) & $\begin{array}{l}\text { Control } \\
\text { Sepsis s/IRA } \\
\text { Sepsis c/IRA }\end{array}$ & $\begin{array}{l}6 \\
4 \\
8\end{array}$ & $\begin{array}{l}6,9 \pm 3,7 \\
5,2 \pm 1,3 \\
6,6 \pm 3,8\end{array}$ & $\begin{array}{l}5,5 \pm 2,2 \\
5,5 \pm 0,6 \\
3,1 \pm 1,8^{*}\end{array}$ & 0,3 \\
\hline Transporte de oxígeno renal (ml/min/kg) & $\begin{array}{l}\text { Control } \\
\text { Sepsis s/IRA } \\
\text { Sepsis c/IRA }\end{array}$ & $\begin{array}{l}6 \\
4 \\
8\end{array}$ & $\begin{array}{l}0,55 \pm 0,2 \\
0,48 \pm 0,1 \\
0,45 \pm 0,1\end{array}$ & $\begin{array}{l}0,46 \pm 0,3 \\
0,43 \pm 0,1 \\
0,29 \pm 0,2\end{array}$ & 0,6 \\
\hline Consumo de oxígeno renal $(\mathrm{ml} / \mathrm{min} / \mathrm{kg})$ & $\begin{array}{l}\text { Control } \\
\text { Sepsis s/IRA } \\
\text { Sepsis c/IRA }\end{array}$ & $\begin{array}{l}6 \\
4 \\
8\end{array}$ & $\begin{array}{l}0,12 \pm 0,04 \\
0,09 \pm 0,01 \\
0,15 \pm 0,08\end{array}$ & $\begin{array}{l}0,09 \pm 0,05 \\
0,11 \pm 0,02 \\
0,08 \pm 0,02\end{array}$ & 0,09 \\
\hline Extracción de oxígeno renal (\%) & $\begin{array}{l}\text { Control } \\
\text { Sepsis s/IRA } \\
\text { Sepsis c/IRA }\end{array}$ & $\begin{array}{l}6 \\
4 \\
8\end{array}$ & $\begin{array}{c}30 \pm 11 \\
29 \pm 5 \\
38 \pm 11\end{array}$ & $\begin{array}{c}27 \pm 8 \\
33 \pm 4 \\
53 \pm 17^{*}\end{array}$ & 0,2 \\
\hline Resistencia vascular renal (mmHg/l/min) & $\begin{array}{l}\text { Control } \\
\text { Sepsis s/IRA } \\
\text { Sepsis c/IRA }\end{array}$ & $\begin{array}{l}6 \\
4 \\
8\end{array}$ & $\begin{array}{l}0,56 \pm 0,2 \\
0,41 \pm 0,1 \\
0,70 \pm 0,2\end{array}$ & $\begin{array}{l}0,59 \pm 0,3 \\
0,44 \pm 0,2 \\
0,72 \pm 0,4\end{array}$ & 0,9 \\
\hline Presión de perfusión renal (PAM-PVR) (mmHg) & $\begin{array}{l}\text { Control } \\
\text { Sepsis s/IRA } \\
\text { Sepsis c/IRA }\end{array}$ & $\begin{array}{l}6 \\
4 \\
8\end{array}$ & $\begin{array}{c}77 \pm 25 \\
60 \pm 8 \\
67 \pm 13\end{array}$ & $\begin{array}{l}76 \pm 28 \\
60 \pm 13 \\
46 \pm 13\end{array}$ & 0,04 \\
\hline
\end{tabular}

Datos presentados como media \pm desviación estándar. $p$-value: Modelo lineal general de medidas repetidas. *Anova de un factor comparando valores finales de una variable entre grupos (significa $p<0,05$ ). 
sépticos sin IRA. Concordantemente, los porcentajes de extracción de oxígeno renales aumentaron en el grupo séptico con IRA $(\mathrm{p}=0,04)$ (Tabla 1$)$.

La presión de perfusión renal $(\mathrm{PPR}=\mathrm{PAM}-$ Presión venosa renal) disminuyó en forma significativa en los animales sépticos con IRA (31\%) respecto de los animales control y sépticos $\sin$ IRA, en los cuales se mantuvo estable $(\mathrm{p}=0,04)$ (Tabla 1). Dado que la presión venosa renal se mantuvo estable y sin diferencia entre los grupos, la caída en la presión de perfusión renal de los animales sépticos con IRA se explica por la caída en la PAM.

\section{Respuesta inflamatoria}

Los niveles de IL-6 aumentaron significativamente sólo en grupo séptico con IRA respecto del grupo séptico sin IRA y del grupo control (C: $109 \pm 21$ a $130 \pm 33 \mathrm{ng} / \mathrm{ml}$, sepsis sin IRA: $101 \pm 12$ a $153 \pm 32 \mathrm{ng} / \mathrm{ml}$ y sepsis con IRA: $210 \pm 150$ a $2.130 \pm 1.547 \mathrm{ng} / \mathrm{ml} ; \mathrm{p}=0,013)$.

\section{Microcirculación renal}

Los animales sépticos con IRA presentaron menores valores de aclaramiento de inulina y de para-aminohipurato (C: $347 \pm 42 \mathrm{ml} / \mathrm{min}$, sepsis sin IRA: $205 \pm 47 \mathrm{ml} / \mathrm{min}$, sepsis con IRA: $89 \pm 74$ $\mathrm{ml} / \mathrm{min} ; \mathrm{p}<0,001)$, lo que refleja una una caída de la tasa de filtración glomerular respecto de los controles de $60 \%$ y una mayor caída en el FPR de aproximadamente $75 \%$. Esto se tradujo en un aumento significativo en la fracción de filtración de ambos grupos sépticos, siendo mayor en el grupo séptico con IRA (C: $24,9 \%$, sepsis sin IRA: 39\%, sepsis con IRA: 49,7\%; $p=0,008)$. La resistencia vascular renal calculada no fue diferente entre animales sépticos y controles (Tabla 1).

Al comparar el FSR medido por doppler, respecto del calculado usando el aclaramiento de paraaminohipurato, se obtiene una correlación significativa para ambos métodos $(\mathrm{p}=0,026$, $\left.\mathrm{R}^{2}: 0,3\right)$.

Tabla 2. Parámetros hemodinámicos sistémicos

\begin{tabular}{|c|c|c|c|c|c|}
\hline Parámetro & Grupo & $\mathbf{n}$ & Basal & $18 \mathrm{~h}$ & Valor-p \\
\hline Presión arterial media $(\mathrm{mmHg})$ & $\begin{array}{l}\text { Control } \\
\text { Sepsis s/IRA } \\
\text { Sepsis c/IRA }\end{array}$ & $\begin{array}{l}6 \\
4 \\
8\end{array}$ & $\begin{array}{l}93 \pm 25 \\
72 \pm 10 \\
86 \pm 12\end{array}$ & $\begin{array}{l}94 \pm 28 \\
77 \pm 12 \\
66 \pm 15\end{array}$ & 0,028 \\
\hline Índice cardiaco (ml/kg/min) & $\begin{array}{l}\text { Control } \\
\text { Sepsis s/IRA } \\
\text { Sepsis c/IRA }\end{array}$ & $\begin{array}{l}6 \\
4 \\
8\end{array}$ & $\begin{array}{c}87 \pm 46 \\
70 \pm 21 \\
110 \pm 39\end{array}$ & $\begin{array}{c}96 \pm 19 \\
102 \pm 36 \\
97 \pm 33\end{array}$ & 0,3 \\
\hline Presión de capilar pulmonar (mmHg) & $\begin{array}{l}\text { Control } \\
\text { Sepsis s/IRA } \\
\text { Sepsis c/IRA }\end{array}$ & $\begin{array}{l}6 \\
4 \\
8\end{array}$ & $\begin{array}{l}14 \pm 2,4 \\
13 \pm 2,5 \\
14 \pm 3,8\end{array}$ & $\begin{array}{l}14 \pm 4,5 \\
15 \pm 2,1 \\
14 \pm 3,4\end{array}$ & 0,3 \\
\hline Lactato arterial (mmol/l) & $\begin{array}{l}\text { Control } \\
\text { Sepsis s/IRA } \\
\text { Sepsis c/IRA }\end{array}$ & $\begin{array}{l}6 \\
4 \\
8\end{array}$ & $\begin{array}{l}0,66 \pm 0,27 \\
0,54 \pm 0,09 \\
0,90 \pm 0,32\end{array}$ & $\begin{array}{c}0,56 \pm 0,15 \\
1,13 \pm 0,21 \\
2,61 \pm 1,83^{*}\end{array}$ & 0,043 \\
\hline Temperatura $\left({ }^{\circ} \mathrm{C}\right)$ & $\begin{array}{l}\text { Control } \\
\text { Sepsis s/IRA } \\
\text { Sepsis c/IRA }\end{array}$ & $\begin{array}{l}6 \\
4 \\
8\end{array}$ & $\begin{array}{l}37,6 \pm 2,3 \\
37,3 \pm 2,6 \\
36,4 \pm 2,7\end{array}$ & $\begin{array}{l}38,2 \pm 1,9 \\
38,1 \pm 1,1 \\
37,8 \pm 3,1\end{array}$ & 0,8 \\
\hline Transporte de oxígeno sistémico (ml/min/kg) & $\begin{array}{l}\text { Control } \\
\text { Sepsis s/IRA } \\
\text { Sepsis c/IRA }\end{array}$ & $\begin{array}{l}6 \\
4 \\
8\end{array}$ & $\begin{array}{c}10,1 \pm 7,5 \\
10,4 \pm 4,4 \\
8,2 \pm 3,2\end{array}$ & $\begin{array}{l}8,4 \pm 2,2 \\
8,1 \pm 2,2 \\
9,2 \pm 3,5\end{array}$ & 0,5 \\
\hline Consumo de oxígeno sistémico (ml/min/kg) & $\begin{array}{l}\text { Control } \\
\text { Sepsis s/IRA } \\
\text { Sepsis c/IRA }\end{array}$ & $\begin{array}{l}6 \\
4 \\
8\end{array}$ & $\begin{array}{l}3,6 \pm 1,8 \\
3,5 \pm 1,0 \\
2,7 \pm 1,3\end{array}$ & $\begin{array}{l}2,8 \pm 1,1 \\
3,3 \pm 1,1 \\
3,6 \pm 1,6\end{array}$ & 0,2 \\
\hline Extracción de oxígeno sistémico (\%) & $\begin{array}{l}\text { Control } \\
\text { Sepsis s/IRA } \\
\text { Sepsis c/IRA }\end{array}$ & $\begin{array}{l}6 \\
4 \\
8\end{array}$ & $\begin{array}{c}39 \pm 8 \\
37 \pm 10 \\
36 \pm 7\end{array}$ & $\begin{array}{c}33 \pm 5 \\
40 \pm 5 \\
43 \pm 18\end{array}$ & 0,3 \\
\hline
\end{tabular}

Datos presentados como media \pm desviación estándar. $p$-value: Modelo lineal general de medidas repetidas. *Anova de un factor comparando valores finales de una variable entre grupos (significa $p<0,05$ ). 


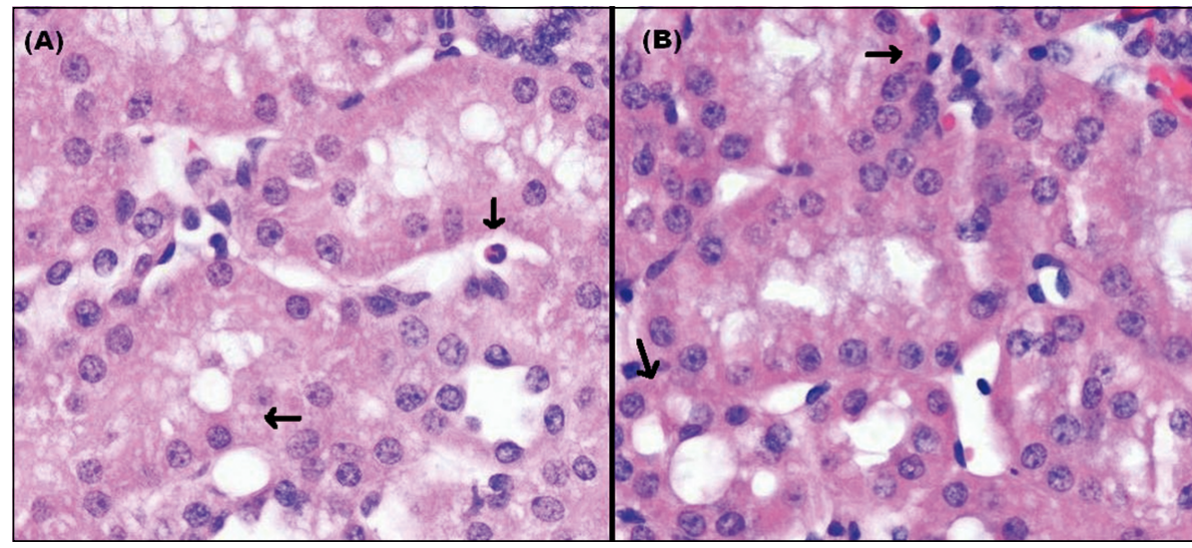

Figura 1. Corte histológico de corteza renal (tinción HE) de animal séptico con IRA. Las flechas señalan infiltración celular y edema celular.

Tabla 3. Variables respiratorias

\begin{tabular}{|c|c|c|c|c|c|}
\hline Parámetro & Grupo & $\mathbf{n}$ & Basal & $18 \mathrm{~h}$ & Valor-p \\
\hline Fracción inspirada de oxígeno (\%) & $\begin{array}{l}\text { Control } \\
\text { Sepsis s/IRA } \\
\text { Sepsis c/IRA }\end{array}$ & $\begin{array}{l}6 \\
4 \\
8\end{array}$ & $\begin{array}{l}26 \pm 3 \\
26 \pm 3 \\
26 \pm 3\end{array}$ & $\begin{array}{l}27 \pm 3 \\
28 \pm 2 \\
29 \pm 4\end{array}$ & 0,028 \\
\hline Presión meseta $\left(\mathrm{cmH}_{2} \mathrm{O}\right)$ & $\begin{array}{l}\text { Control } \\
\text { Sepsis s/IRA } \\
\text { Sepsis c/IRA }\end{array}$ & $\begin{array}{l}6 \\
4 \\
8\end{array}$ & $\begin{array}{l}15 \pm 3 \\
17 \pm 2 \\
16 \pm 2\end{array}$ & $\begin{array}{l}16 \pm 2 \\
17 \pm 2 \\
18 \pm 4\end{array}$ & 0,1 \\
\hline Compliance estática $\left(\mathrm{ml} / \mathrm{cmH}_{2} \mathrm{O}\right)$ & $\begin{array}{l}\text { Control } \\
\text { Sepsis s/IRA } \\
\text { Sepsis c/IRA }\end{array}$ & $\begin{array}{l}6 \\
4 \\
8\end{array}$ & $\begin{array}{c}34 \pm 12 \\
30 \pm 5 \\
32 \pm 5\end{array}$ & $\begin{array}{l}29 \pm 6 \\
26 \pm 5 \\
27 \pm 8\end{array}$ & 0,3 \\
\hline
\end{tabular}

Datos presentados como media \pm desviación estándar. p-value: Modelo lineal general de medidas repetidas.

\section{Histología}

El análisis histológico mostró que ninguno de los grupos presentó necrosis significativa, pero en el grupo séptico con IRA se observó infiltración celular y edema celular (Figura 1).

\section{Variables respiratorias}

La Tabla 3 resume las principales variables respiratorias. Se observa que los cambios en la $\mathrm{FiO}_{2}$, presión meseta y "compliance" estática no fueron significativos ni diferentes entre los grupos de estudio.

\section{Discusión}

El objetivo de este trabajo fue evaluar mecanismos relevantes en la génesis de IRA en las etapas precoces de la sepsis. Nuestra hipótesis inicial su- gería que los cambios en el gasto cardiaco y presión arterial (macro-hemodinamia) no eran mecanismos relevantes mientras se mantuviese una PAM sobre $60 \mathrm{mmHg}$. Por el contrario, planteamos que cambios en el FSR y en la fracción de filtración gatillados por fenómenos locales inflamatorios eran mecanismos de mayor relevancia. Sin embargo, de acuerdo a los resultados observados, es posible concluir que los mecanismos que participan en el desarrollo de IRA durante las etapas precoces de la sepsis severa son múltiples y actúan en forma sinérgica, y que la caída en la presión arterial es un mecanismo preponderante.

De los elementos medidos para explicar el desarrollo de IRA en las etapas precoces de la sepsis, aquellos animales sépticos que presentaron IRA tuvieron una caída significativa de la PAM y del FSR. Considerando que tanto la RVR como la PVR se mantuvieron estables, cabe concluir que el 
principal determinante en el descenso del FSR es la disminución significativa de la PPR, explicada por la disminución de la PAM.

La caída en el FSR asociado a una caída en PPR aparece como el principal determinante en la génesis de IRA en nuestro estudio. La importancia de la caída en la presión de perfusión renal como mecanismo de IRA ha sido destacada previamente por otros autores ${ }^{16,17}$. En particular, el estudio de Chvojka et al. ${ }^{16}$ muestra que en animales sépticos, la existencia de IRA se correlacionó principalmente a una disminución en la presión de perfusión renal.

En nuestro estudio, el descenso en la PPR y del FSR no se asociaron a necrosis celular significativa de acuerdo al análisis histológico. Por el contrario, parece existir un aumento en la tasa de metabolismo anaeróbico y aeróbico como lo sugiere el aumento en la extracción de oxígeno renal y la mayor producción de lactato renal. Estos resultados sugieren que la oxigenación renal, aunque disminuida, es suficiente y no explica por si sola el desarrollo de IRA en la sepsis. Tradicionalmente la hipoperfusión renal, asociada a necrosis celular (necrosis tubular aguda) ha sido considerada como la causa primaria de la IRA en la sepsis. Sin embargo, la mayoría de los datos que sugieren una etiología isquémica de la IRA en la sepsis provienen de estudios de los años 80 realizados en modelos animales de isquemia y reperfusión (e.g. clampeo de la arteria renal $)^{18}$. Tales modelos producen una fisiología completamente distinta en comparación con la fisiopatología de la sepsis, en que se observa un gasto cardiaco elevado y una resistencia vascular sistémica significativamente disminuida. Nuestro estudio confirma por un lado la importancia de la caída de la PPR y del FSR como mecanismos de IRA, pero sugiere que otros mecanismos no isquémicos participan también en la genesis de IRA en la sepsis. De esta manera, nuestros resultados están de acuerdo con varios otros estudios recientes, que muestran que la IRA durante la sepsis se desarrolla sin necrosis renal significativa $a^{7,19,20}$.

El aumento observado en la fracción de filtración en los animales sépticos con IRA se explica por un descenso significativamente mayor del FPR respecto del descenso observado en la tasa de filtración glomerular (FG). La caída en el FPR se explica mayormente por la caída en el FSR, pero la asimetria en el descenso de la FG y el FPR pudiera explicarse por algún grado de vasocontricción microvascular renal, que no se refleja en los valores de RVR donde sólo se considera la PAM y la PVR para su cálculo. La vasocontricción microvascular renal ha sido descrita previamente como un mecanismo de IRA en la sepsis ${ }^{21,22}$. Esta vasocontricción ha sido explicada por activación del sistema de renina -angiotensina y por un aumento de la actividad nerviosa simpática renal ${ }^{23,24}$.

La respuesta inflamatoria característica de la sepsis, se ha estudiado como un mecanismo de IRA en la sepsis ${ }^{25,26}$. Nuestro estudio demostró un marcado aumento en los niveles de IL-6 en animales sépticos con IRA, lo cual no sólo es un marcador de inflamación, sino también un índice del daño celular mediado por la respuesta inmune. Los riñones parecen ser particularmente vulnerables a la lesión mediada por citoquinas, dada la asociación persistente entre niveles de inflamación y el desarrollo de IRA ${ }^{27}$.

Nuestro estudio tiene limitaciones. Se estudió un número pequeño de animales por grupo, por lo tanto, existe el riesgo de error tipo II, sin embargo, esto se hace en un intento de reducir el daño animal. No se midieron marcadores de daño tisular como pudiesen ser estrés oxidativo tisular, función mitocondrial o microcirculación por punción.

En conclusión, nuestro estudio sugiere que los mecanismos que participan en el desarrollo de IRA en las etapas precoces de la sepsis son múltiples y actuan sinérgicamente. La caída de la PPR y del FSR son mecanismos centrales, aunque no se asocian a necrosis celular, por lo que otros mecanismos, como la inflamación sistémica y la vasocontricción microvascular, pudiesen contribuir al desarrollo de IRA. Es necesario desarrollar más estudios para identificar y tratar factores potencialmente prevenibles o reversibles de IRA en la sepsis.

\section{Referencias}

1. Abraham E, Singer M. Mechanisms of sepsis-induced organ dysfunction. Crit Care Med 2007; 35 (10): 240816.

2. Angus DC, Linde-Zwirble WT, Lidicker J, Clermont G, Carcillo J, Pinsky MR. Epidemiology of severe sepsis in the United States: analysis of incidence, outcome, and associated costs of care. Crit Care Med 2001; 29 (7): 1303-10. 
Determinantes precoces de injuria renal en la sepsis abdominal - T. Regueira et al

3. Vincent JL, Sakr Y, Sprung CL, Ranieri VM, Reinhart K, Gerlach H, et al. Sepsis in European intensive care units: results of the SOAP study. Crit Care Med 2006; 34 (2): 344-53.

4. Ostermann M, Chang RW. Acute kidney injury in the intensive care unit according to RIFLE. Crit Care Med 2007; 35 (8): 1837-43; quiz 1852.

5. Langenberg C, Bellomo R, May C, Wan L, Egi M, Morgera S. Renal blood flow in sepsis. Crit Care 2005; 9 (4): R363-74.

6. Regueira T, Andresen M, Mercado M, Downey P. Physiopathology of acute renal failure during sepsis. Medicina Intensiva 2011; 35 (7): 424-32.

7. Hotchkiss RS, Swanson PE, Freeman BD, Tinsley KW, Cobb JP, Matuschak GM, et al. Apoptotic cell death in patients with sepsis, shock, and multiple organ dysfunction. Crit Care Med 1999; 27 (7): 1230-51.

8. Takasu O, Gaut JP, Watanabe E, To K, Fagley RE, Sato $\mathrm{B}$, et al. Mechanisms of cardiac and renal dysfunction in patients dying of sepsis. Am J Respir Crit Care Med 2013; 187 (5): 509-17.

9. Benes J, Chvojka J, Sykora R, Radej J, Krouzecky A, Novak I, et al. Searching for mechanisms that matter in early septic acute kidney injury: an experimental study. Crit Care 2011; 15 (5): R256.

10. Legrand M, Bezemer R, Kandil A, Demirci C, Payen D, Ince $\mathrm{C}$. The role of renal hypoperfusion in development of renal microcirculatory dysfunction in endotoxemic rats. Intensive Care Med 2011; 37 (9): 1534-42.

11. Tran M, Tam D, Bardia A, Bhasin M, Rowe GC, Kher A, et al. PGC-1alpha promotes recovery after acute kidney injury during systemic inflammation in mice. J Clin Invest 2011; 121 (10): 4003-14.

12. de Geus HR, Bakker J, Lesaffre EM, le Noble JL. Neutrophil gelatinase-associated lipocalin at ICU admission predicts for acute kidney injury in adult patients. Am J Respir Crit Care Med 2011; 183 (7): 907-14.

13. Cruz DN, de Cal M, Garzotto F, Perazella MA, Lentini $\mathrm{P}$, Corradi V, et al. Plasma neutrophil gelatinase-associated lipocalin is an early biomarker for acute kidney injury in an adult ICU population. Intensive Care Med 2010; 36 (3): 444-51.

14. Shapiro NI, Trzeciak S, Hollander JE, Birkhahn R, Otero $\mathrm{R}$, Osborn TM, et al. The diagnostic accuracy of plasma neutrophil gelatinase-associated lipocalin in the prediction of acute kidney injury in emergency department patients with suspected sepsis. Ann Emerg Med 2010; 56 (1): 52-59 e51.
15. Uchino S, Bellomo R, Goldsmith D, Bates S, Ronco C. An assessment of the RIFLE criteria for acute renal failure in hospitalized patients. Crit Care Med 2006; 34 (7): 1913-7.

16. Chvojka J, Sykora R, Krouzecky A, Radej J, Varnerova V, Karvunidis T, et al. Renal haemodynamic, microcirculatory, metabolic and histopathological responses to peritonitis-induced septic shock in pigs. Crit Care 2008; 12 (6): R164.

17. Leblanc M, Kellum JA, Gibney RT, Lieberthal W, Tumlin J, Mehta R. Risk factors for acute renal failure: inherent and modifiable risks. Curr Opin Crit Care 2005; 11 (6): 533-6.

18. Wan L, Bellomo R, Di Giantomasso D, Ronco C. The pathogenesis of septic acute renal failure. Curr Opin Crit Care 2003; 9 (6): 496-502.

19. Langenberg C, Bagshaw SM, May CN, Bellomo R. The histopathology of septic acute kidney injury: a systematic review. Crit Care 2008; 12 (2): R38.

20. Langenberg C, Wan L, Egi M, May CN, Bellomo R. Renal blood flow in experimental septic acute renal failure. Kidney Int 2006; 69 (11): 1996-2002.

21. Schrier RW, Wang W. Acute renal failure and sepsis. N Engl J Med 2004; 351 (2): 159-69.

22. Boffa JJ, Arendshorst WJ. Maintenance of renal vascular reactivity contributes to acute renal failure during endotoxemic shock. J Am Soc Nephrol 2005; 16 (1): 117-24.

23. Wang W, Falk SA, Jittikanont S, Gengaro PE, Edelstein CL, Schrier RW. Protective effect of renal denervation on normotensive endotoxemia-induced acute renal failure in mice. Am J Physiol Renal Physiol 2002; 283 (3): F583-7.

24. Tarpey SB, Bennett T, Randall MD, Gardiner SM. Differential effects of endotoxaemia on pressor and vasoconstrictor actions of angiotensin II and arginine vasopressin in conscious rats. Br J Pharmacol 1998; 123 (7): 1367-74.

25. Abraham E. Neutrophils and acute lung injury. Crit Care Med 2003; 31 (4 Suppl): S195-9.

26. Marshall JC, Vincent JL, Fink MP, Cook DJ, Rubenfeld G, Foster D, et al. Measures, markers, and mediators: toward a staging system for clinical sepsis. A report of the Fifth Toronto Sepsis Roundtable, Toronto, Ontario, Canada, October 25-26, 2000. Crit Care Med 2003; 31 (5): 1560-7.

27. Kikeri D, Pennell JP, Hwang KH, Jacob AI, Richman $\mathrm{AV}$, Bourgoignie JJ. Endotoxemic acute renal failure in awake rats. Am J Physiol 1986; 250 (6 Pt 2): F1098-106. 